\title{
Intrathyroid Abscess Formation with Multiple Necrotizing Cervical Lymphadenitis due to Meticillin Resistant Staphylococcus aureus (MRSA); ACase Report with Literature Review Senanayake KJ, Pitigalaarachchi PR
}

Department of Surgery, Faculty of Medicine and Allied Health Sciences, Rajarata University of Sri Lanka ${ }^{1}$, Department of General Surgery, Teaching Hospital Kandy, Sri Lanka ${ }^{2}$,

\begin{abstract}
\section{Introduction}

Intrathyorid abscess formation due to MRSA is rare. It needs high index of suspicion to diagnose early. Early diagnosis and treatment improves the outcome.
\end{abstract}

\section{The Patient}

A 77 year old Sri Lankan female, presented to the emergency surgical department with a tender swelling in the anterior neck. On examination there was an enlarged thyroid gland with multiple cervical lymphadenopathy. Ultrasound scan revealed an abscess within the right lobe of the thyroid. Ultrasound guided aspiration of the puss and culture ABST done and the MRSA was isolated after 24 hour incubation. Incision and drainage of the abscess was done. Intravenous antibiotics were started according to the culture ABST.

\section{Conclusion}

It needs high index of suspicion to diagnose the thyroid abscess early. Ultrasound guided aspiration, Incision and drainage and early administration of sensitive antibiotic would improve the outcome.

\section{Introduction}

Abscess formation within the thyroid gland is rare (1). Because of its rarity, it is often not suspected and hence the diagnosis would be delayed. Possibility of thyroid abscess formation should be suspected in any patient who presented with tender neck swelling in thyroid area.

\section{The patient}

A seventy seven year old Sri Lankan female patient presented to the emergency surgical department of Teaching Hospital Kandy, Sri Lanka with high fever and neck swelling for 2 days. She did not have difficulty in breathing. The onset of fever was gradual and associated with chills. Relatives noticed that the patient was confused and disoriented. On examination, the patient was dehydrated and drowsy. There was a large right sided tender, warm swelling in the thyroid region with multiple cervical lymphadenopathy.

An urgent white cell count showed a very high leucocyte count with neutrophilia. The ultrasound scan revealed an abscess in the right lobe of the thyroid gland with multiple cervical lymphadenopathy. A CT scan (figure 1.) of the neck with reconstruction was performed to assess the extension. Ultrasound guided aspiration of the abscess was done and pus was sent for culture and antibiotic sensitivity (table 1). The gram stain showed few gram negative bacilli and few gram positive bacilli. Pus grew Methicillin Resistant Staphylococcus aureus (MRSA) after 24 hours of incubation. The patient was also screened for tuberculosis and it was negative. The Biopsy of cervical lymph node showed necrosis with secondary deposit from a sqamous cell carcinoma of which the primary was not found. She was a known patient with diabetic mellitus and had poor control of blood sugar. The patent was rehydrated with intravenous normal saline and adequate urine output was maintained. After taking the puss for culture ABST by USS guided aspiration the patient was empirically treated with cefuroxime $750 \mathrm{mg}$ 
eight hourly and the patient was treated with oral fucidic acid and intravenous vancomycin after the culture report. Incision and drainage was performed under general anesthesia. Patent had a good recovery from the sepsis after post operative day 3 . Informed written consent was taken from the patient for photography and publishing the case report.

Table 1. Sensitivity pattern of MRSA from the pus culture

\begin{tabular}{|l|l|}
\hline Antibibiotic & Sensitivity \\
\hline Cefuroxime & Resistant \\
\hline Clindamycin & Resistant \\
\hline Cloxacillin & Resistant \\
\hline Co-trimoxazole & Resistant \\
\hline Erythromycin & Resistant \\
\hline Fusidic acid & Sensitive \\
\hline Vancomycin & Sensitive \\
\hline
\end{tabular}

\section{Discussion}

Abscess formation within the thyroid gland is rare (1). The intrathyroid abscess formation with MRSA may be rarer. The oldest case of thyroid abscess reported in the Pub Med was in 1894 by Ransohoff J (2). There are 319 cases reported in English in the literature from 1900 to 2000 , et al and Jacobs et al (3).Possible causes of infection of thyroid gland are either direct inoculation or hematological spread. The commonest route of infection is probably hematological (3). Other possible causes reported are the direct trauma such as, FNAC, esophageal perforation due to fish bone injury and in children's it is commonly due toanatomical anomalies such as piriform sinus fistula $(1,4,5)$.The acute suppurative thyroiditis now rarely progress to thyroid abscess formation due to widespread use of antibiotics (6). The hemorrhage in to a thyroid cyst leading to secondary infection is a possible cause for the intrathyroid abscess formation. Rarely the post anginal sepsis (Lemierre's Syndrome) could present with thyroid abscess (7) and further it has been reported secondary to biliary sepsis (8).The bacteraemia cause by the MRSA is reported in immunologically compromised patients (9).The thyroid abscess formation commonly reported among the immunocompromised (1). In the present case the patient is immunologically compromised due to the presence of diabetes mellitus and the underling malignancy of which the primary is unknown.

The rarity of abscess formation in thyroid leads to delay in the diagnosis due to the fact that it is not included in the differential diagnosis list in many physicians. The thyroid gland is highly resistant to be infected. The resistance of thyroid gland to form abscess is multifactorial. Presence of a capsule, high concentration of iodine, very high vascularity, and presence of high lymphatic drainage was postulated (1).

The commonest organism of thyroid abscess formation reported is staphylococci aurius (3). Butthe reported cases of thyroid abscess due to MRSA are minimal. The other organisms that have been isolated and responsible for abscess formation in the thyroid are tuberculosis, norcadia, E.coli, Salmonella, Eikenella corrodens, Actinomycosis Rhodoccus equi, and fugal infections suchas Cryptococcus and aspergillous etc.

Cases of thyoiditis with thyrotoxicosis cause bybacteria are reported (10). Therefore the altered thyroid function must be anticipated and suspected in a patient with thyroid abscess. However the current case the patient is euthyroid.

The diagnosis of the thyroid abscess is straightforward if it is suspected and examine. Clinically the enlarged, tender thyroid with the evidence of inflammation of overlying skin, multiple cervical lymphadinopathy gives a clue to the diagnosis. Ultrasonogrphy gives details about the abscess cavity, cervical lyphadenophathy and the diagnosis can be 


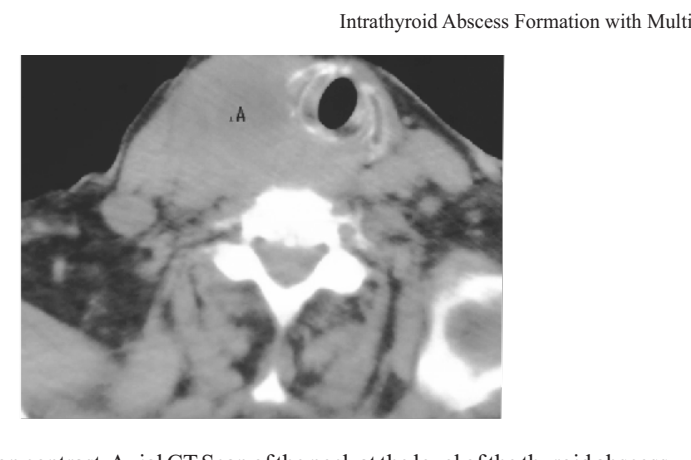

Figure 1. Non contrast, Axial CT Scan of the neck at the level of the thyroid abscess

Contrast enhanced CT scan also delineates the anatomical extension of the thyroid abscess and may be helpful to plan the management. In the current case a plain CT scan (figure 1.) was donedue topresence of multiple allergies. Barium swallow can be used to assess theanatomical abnormalities after inflammatory process is resolved $(11,12)$.

The usual treatment includes intervention of the ENT service with incision and drainage of theabscess or partial thyroidectomy, depending upon the presence or absence of underlying thyroid pathologies, together with intravenous antibiotics (5).In the current case the treatment was a high dose ofsensitive intravenous antibiotics and ultrasound guided aspiration together with incision anddrainage. The most frequently recommended treatment in the literature is surgery either excisionor incision and drainage (3). However the role of more extensive surgery such as total or neartotal thyroidectomy in the management of intrathyroid abscess is not widely discussed. Non surgical management with ultrasound guided aspiration is suggested (13).However there is a possibility of recollection.

\section{Conclusion}

Abscess formation due to MRSA within the thyroid gland is rare. Thyroid abscess should besuspected in immunocompromised patients presenting with neck pain and swelling. Thiswould enable early diagnosis and treatment

\section{References}

1. Herndon MD, Christie DB, Ayoub MM, Duggan AD. Thyroid abscess: case report andreview of the literature. Am Surg. 2007 Jul;73(7):725-8.

2. Ransohoff J.Thyroid Abscess; Thyroidectomy; Recovery. Ann Surg. 1894 Oct;20(4):406-13.

3. Jacobs A. Gros DC, Gradon JD. Thyroid abscess due to Acinetobacter cakoaceticus: Casereport and review of the causes of and current management strategies of thyroidabscesses. South Med J 2(X) 3:96:300-7.

4. Lin ZH, Teng YS, Lin M. Acute thyroid abscess secondary to esophageal perforation. JInt Med Res. 2008 JulAug;36(4):860-4.

5. Stavreas NP, Amanatidou CD, Hatzimanolis EG, Legakis I, Naoum G,Lakka-Papadodima E, Georgoulias G, Morfou P, Tsiodras S. Thyroid abscess due to a mixedanaerobic infection with Fusobacterium mortiferum. J Clin Microbiol. 2005Dec;43(12):6202-4.

6. Menegaux, F., G. Biro, C. Schatz, and J. P. Chigot. 1991. Thyroid abscess. Apropos of 5 cases. Ann. Med. Interne (Paris). 142:99-102

7. Kara E, Sakarya A, KeleÅŸ C, Borand H et al. Case of Lemierre's syndrome presentingwith thyroid abscess. Eur J Clin Microbiol Infect Dis. 2004 Jul;23(7):570-2.

8. Mathew J, Goodfellow P, Chadwick DR. Thyroid abscess: an unusual case secondary tobiliary sepsis. Hosp Med. 2003 Oct;64(10):622-3.

9. Mitchell DH, Howden BP. Diagnosis and management of Staphylococcus aureusbacteraemia. Intern Med J. 2005 Dec;35 Suppl 2:S17-24. 
10. Al-Kordi RS, Alenizi E, Elgazzar AH. Acute suppurative thyroiditis with abscess, gasformation, and thyrotoxic crisis. Nuklearmedizin. 2008;47(4):N446.

11. Houghton DJ, Gray HW, MacKenzie K. The tender neck:Thyroiditis or thyroid abscess?Clin Endocrinol (Oxf) 1995:48:5214.32.

12. Takai SI. Miyauchi A, Matsuzuka F. et al. Intemal fistula as a route of infection in acutesuppurative thyroiditis. Lancet 1979:1:751-2.

13. Ilyin A, Zhelonkina N, Severskaya N, Romanko S. Nonsurgical management of thyroidabscess with sonographically guided fine needle aspiration. J ClinUltrasound. $2007 \mathrm{Jul}$ Aug;35(6):333-7.. 